\title{
Die Macht in der Psychiatrie
}

Michael Kammer-Spohn

Korrespondenz:

Michael Kammer-Spohn

Facharzt für Psychiatrie und

Psychotherapie

Leitender Arzt Allgemeine

Psychiatrie St. Gallische

Psychiatrie-Dienste Süd

Klinik St. Pirminsberg

Klosterweg

$\mathrm{CH}-7312$ Pfäfers

michael.kammer[at]psych.ch

\section{Vorüberlegungen}

Die Macht der Gesellschaft erreicht den gesunden wie auch kranken individuellen Menschen sehr einschneidend, sobald er in seinem Verhalten die Schwelle der Normalität unter- oder überschreitet. Wenn die gesellschaftlich-kulturellen oder auch gesetzlichen Grenzen verletzt werden und dafür ein krankhafter Prozess verantwortlich gemacht wird, wird die Disziplinargewalt der Gesellschaft an das medizinische System übertragen. Das medizinische System ist durch Gesetze legitimiert, Macht auszuüben. Diese schützen vor Willkür und Bestrafung und haben die Behandlung mit gesundheitlicher Wiederherstellung zum Ziel sowie insbesondere in der psychiatrischen Behandlung auch den Schutz von Drittpersonen. In der Psychiatrie werden Menschen behandelt, die teilweise definitiv die Behandlung verweigern. Dies ist anders als in der somatischen Medizin, wo es selbstverständlich erscheint, dass eine Notfallbehandlung auch ohne Einwilligung erfolgen muss, etwa wenn der Patient bewusstlos ist. Die Machtausübung der Psychiatrie steht immer wieder in der Kritik der Öffentlichkeit: Menschen werden zwangseingewiesen, zwangsbehandelt oder müssen Medikamente nehmen, die angeblich ihre Persönlichkeit verändern. Andrerseits wird die Psychiatrie angegriffen, sobald von einem Menschen mit einer psychischen Krankheit ein Unfall oder gar eine Straftat ausgeht. Dann werden eine scheinbar mangelnde Überwachung, eine fehlende Zwangsbehandlung oder fehlende Medikamente beklagt. Wo die Grenzen zu Zwang und Gewalt festzulegen sind, ist schwer zu bestimmen und soll hier nicht näher erörtert werden. Macht wird in der Psychiatrie von professionell ausgebildeten Menschen ausgeübt, wobei Ärzte letztverantwortlich sind bei dieser fachlich und ethisch anspruchsvollen Aufgabe. Die Psychiatrie ist immer wieder neu gefordert, Möglichkeiten zu finden, die ihr über die Kranken übertragene Macht mit dem notwendigen, aber minimal möglichen Mass an Zwang und Gewalt auszuüben. Wie verhält es sich nun mit der Macht der Psychiatrie und in der Psychiatrie? Ist es abzulehnen, dass Menschen unter diese Macht gestellt werden oder müsste im Gegenteil die Gesellschaft vor den psychisch kranken Menschen besser geschützt werden? Und wer tut sich das an, im psychiatrischen System zu arbeiten, wenn man sich ständig mit dieser Problematik auseinandersetzen muss, was hilft dabei? Diese Fragestellungen möchte ich im folgenden Essay näher beleuchten.

\section{Die Macht psychisch Kranker}

Psychisch kranke Menschen üben Macht aus. Dabei sind nicht nur offensichtliche Drohungen oder Ge-

\section{Pouvoir et psychiatrie}

La psychiatrie fait non seulement partie de la médecine sur le plan médical et scientifique, mais elle est aussi ancrée dans la société et notamment dans le système juridique, qui la mandate et la légitime. En psychiatrie, les patients entrent parfois, en raison de leur maladie, en conflit avec les autres et la société, ce qui fait qu'on les pousse ou les contraint à suivre un traitement. Or ce n'est pas comme en justice où la présomption d'innocence est applicable. II ne faut pas oublier que le problème se situe en premier lieu dans l'environnement de la personne atteinte d'une maladie psychique et que c'est le seul motif de consultation. Ce n'est qu'après, au moment du traitement, que la question de l'exercice du pouvoir se pose. Malgré tous les progrès réalisés dans la recherche des causes et le traitement des maladies psychiques, le conflit entre individu et société demeure. Gérer les conflits de pouvoir de manière appropriée et éthique, tant dans des cas individuels que sur le plan structurel, contribue grandement au succès du traitement psychiatrique.

waltanwendungen gemeint. Die Macht der psychisch Kranken beeinflusst das «Miteinandersein» mit anderen Menschen anders als die Macht der Gesunden. Letztere stützt sich auf gesunde Grenzen zu den Mitmenschen. Von diesen nehmen wir an, dass sie vernünftig, also frei, eigenverantwortlich und rücksichtsvoll, aber auch transparent und nachvollziehbar entscheiden können.

Menschen mit Depressionen leben in einem Gefühl der Ohnmacht, nach Martin Heidegger in einer Daseins-Verfassung der Angst und des fehlenden Vollzugs. Ein depressiver Mensch glaubt, dass sich niemals etwas zum Guten ändern wird. Wenn kein Sinn im Dasein gefunden wird, kann als logische Konsequenz des subjektiv ausweglosen Dahinvegetierens oft in einem letzten Akt von Autonomie eine 


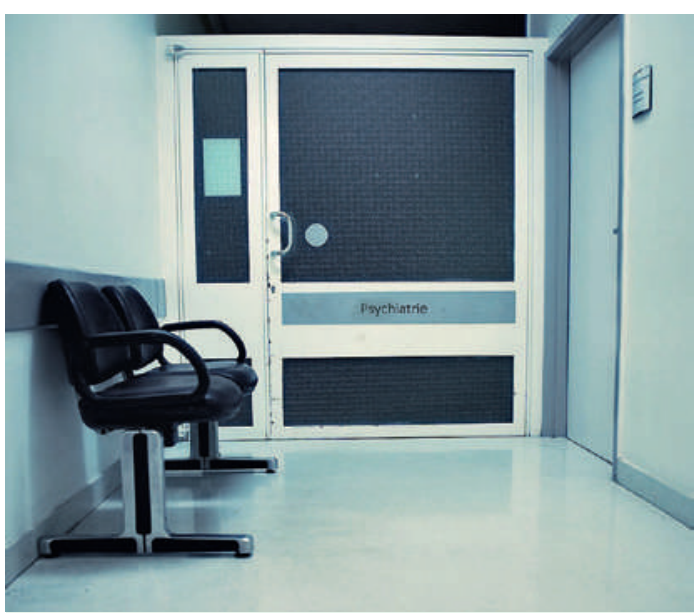

Entscheidend in der Psychiatrie ist das «Wie viel» und das «Wie» der Machtausübung über den kranken Menschen.

Suizidabsicht entstehen. Ein Mensch in einer schweren Depression muss daher vor diesem Akt der Selbstvernichtung geschützt werden, insbesondere wenn wir die Depression als vorübergehende und behandelbare Krankheit begreifen.

Anders verhält es sich bei einem Menschen, der die Symptome einer Manie zeigt: Dieser Mensch ist von seiner Allmacht überzeugt. Primär sind zuerst die Grenzen, die er sich in seinem eigenen Lebensvollzug setzt, und dann die normalen Grenzen zum Mitmenschen verschwunden. So verprasst er sein Vermögen, verhält sich unmoralisch, entwickelt tausend Ideen, beginnt Projekte und Vorhaben, die zuvor in seinem Leben niemals denkbar und schon gar nicht realisierbar waren. Oft hat er eine eigenartige, charismatische Ausstrahlung auf Mitmenschen, weiss diese zu begeistern und zu unterhalten. Vor allem aber - ähnlich wie beim Depressiven - zeigt sich ein Bruch in der Kontinuität seines Lebens oder Daseins. Nicht umsonst hat Kraepelin als wichtiges Kriterium der Diagnostik psychischer Störungen den Verlauf in einem Zeitkontinuum eingeführt.

Auch Menschen, die an Schizophrenie oder schizophrenie-ähnlichen Psychosen erkranken, üben oft Macht auf ihre Umgebung aus. Dadurch, dass sie eine eigene, unverständliche und meist unteilbare Subjektivität erleben, wird diese Machtausübung problematisch. Beispielsweise eine Frau, die mit Engeln kommuniziert und auf innere Stimmen hört, zwingt ihren Mann und ihre Kinder, sie zu versorgen und ihr in ihrer Subjektivität nicht zu nahe zu treten. Sie kann ihre Rolle nicht mehr erfüllen, sei es als Mutter oder als Partnerin. Dadurch, dass sie nicht konsensfähig ist, sondern unverrückbar in ihrem «verrückten» Erleben festhält, ergeben sich zwingend Konflikte. Ein anderer an Schizophrenie erkrankter Mensch verhält sich vielleicht unangemessen am Arbeitsplatz und bringt Abläufe durcheinander. Auch dabei hat er Macht und Einfluss auf seine Arbeitskollegen und auf die Vorgesetzten wie auch auf das Ergebnis der Arbeit. Weitere Beispiele für eine «stö- rende» Machtausübung sind uns allen bekannt bei Menschen mit Demenz, bei Persönlichkeitsstörungen oder bei Abhängigkeitserkrankungen.

\section{Die Macht der Behandler}

Die Machtproblematik lässt sich aus verschiedenen Perspektiven beleuchten: aus jener der Behandler, derjenigen der betroffenene Menschen, aus jener der Angehörigen und nicht zuletzt jener der Gesellschaft. Primär geht es um einen krankhaften Prozess, der erkannt wird und gebessert werden kann. Dieser hat sich des Patienten «bemächtigt», und der Patient hat Anspruch auf Behandlung und Heilung. Dann sind es auch Angehörige des Kranken, die Nachbarn oder die Arbeitgeber, die Interesse an der Behandlung haben - all die, die sich der Macht des Kranken ausgeliefert fühlen. Und dann sind es Strukturen der Gesellschaft, die Aufträge zur Behandlung ableiten lassen: Die öffentliche Ordnung und Sicherheit sowie die geforderte Teilhabe an Arbeitsprozess und gesellschaftlicher Verantwortung. Unsere Gesellschaft funktioniert nur, wenn die Mehrzahl der Regeln akzeptiert und befolgt wird. Menschen, die nicht arbeiten, bekommen Arbeitslosengeld, Sozialhilfe oder Rente zum Überleben. Menschen, die krank oder behindert sind, erhalten eine Rente, wenn sie nicht arbeiten. Menschen, die als faul oder träge attribuiert werden, erhalten Sozialhilfe, oftmals mit Auflagen. Auch im Strafrecht herrscht die Unterteilung in krank oder gesund. Straftatbestände, die im Rahmen einer psychischen Störung oder Krankheit verübt werden, werden milder gebüsst. Hier zielt die Ausübung der gesellschaftlichen Macht weniger auf das straffällige Individuum und mehr auf dessen Krankheit.

Die Behandlung der Krankheit erfordert vom kranken Menschen seine Anwesenheit im Behandlungsprozess. Er muss Gespräche mit Psychologen führen, also seine Zeit diesem unterwerfen; er muss sich in einer psychiatrischen Klinik aufhalten, sich überwachen oder auch einsperren lassen, manchmal lebenslänglich; und er muss Eingriffe in seine körperliche Unversehrtheit hinnehmen, Medikamente schlucken, sich Injektionen verabreichen lassen, zum diagnostischen Prozess beitragen. Aus der Perspektive der Betroffenen bedeutet das «Unter-dieMacht-der-Psychiatrie-gestellt-sein» das AusgeliefertWerden an eine fremde, medizinische Macht. Die zuvor noch erlebte Selbstwirksamkeit wird ihnen entzogen. Es ist für sie ein unverständlicher Übergriff in die eigene Subjektivität. Psychisch kranke Menschen, insbesondere solche mit Schizophrenie, können oft nicht mehr mit anderen kooperieren, da sie weder nonverbale noch verbale Signale vernünftig deuten können. Viele Menschen erachten jedoch, so lange sie gesund sind, die Macht der Psychiatrie auch für sich selbst im Falle einer Erkrankung als notwendig, beispielsweise um ihre Kinder vor den Folgen eines eigenen Suizids zu schützen. Oft wird auch von den Betroffenen später, nach erfolgreich verlaufener Be- 
handlung, rückblickend diese Machtausübung gutgeheissen oder wenigstens akzeptiert.

\section{Das Mass der Machtausübung}

Manchmal ist Behandlung nicht ohne ein gewisses Mass an Machtausübung, Zwang oder Gewalt möglich. Entscheidend und auch modifizierbar ist das «Wie viel» und das «Wie» der Machtausübung über den kranken Menschen. Früher waren es Familienangehörige oder die Dorfgemeinschaft, heute sind es Gesetze, Gerichte und Ärzte des medizinischen Systems, denen die Macht über den «Verrückten» übertragen wird. Heute besteht weniger Willkür, es gibt transparente Kriterien und Regeln, aber doch üben Menschen über andere Macht und Gewalt aus. Damit eine erzwungene Behandlung erfolgversprechend sein kann, sollte sie immer zwei Perspektiven beinhalten: Sie muss im Sinne des Kranken und im haltens von der Gesellschaft ausgesondert werden, benötigen die professionellen Helfer genügend Freizeit, in der sie Normalität erleben können und ihre eigenen Grenzen «eichen» können. Für die Behandler ausserdem wichtig sind die klaren externen Grenzen, wie sie durch Gerichtsbeschlüsse oder Vorgaben von der Justiz vermittelt werden. Um die Machtausübung in der Psychiatrie auf weniger Zwang und noch weniger Gewalt zu reduzieren, sind auch äusserliche Faktoren sehr wichtig. Patienten benötigen eine stressarme und räumlich grosszügige Umgebung mit Rückzugsmöglichkeiten. Es muss Vertrauen in sie selbst und in die Mitmenschen entstehen können. Die Betroffenen müssen sich wertgeschätzt fühlen und schrittweise mehr Eigenverantwortung aufnehmen können. Überfüllte, enge und laute Behandlungsstationen mit gestresstem Personal verhindern oder verzögern effektive Behandlungen. Auch ambulante Behandlungs-

\section{«Manchmal ist Behandlung nicht ohne ein gewisses Mass an Machtausübung, Zwang oder Gewalt möglich.»}

Sinne des Schutzes der Gesellschaft erfolgen. Mitarbeiter der psychiatrischen Institutionen, die diese Macht legitimiert ausüben, müssen klaren und hohen Kriterien genügen: Die Behandler müssen sich ihrer Doppelfunktion - Behandler der Patienten wie auch verantwortlich zu sein für Angehörige und Gesellschaft - bewusst sein.

Es werden nicht alle Menschen gesund oder erreichen wieder eine vollständige «Normalität» mit Teilhabe und Verantwortung im gesellschaftlichen Kontext. Zur Behandlung gehört zwingend, dass realistische Ziele verfolgt werden, dass bei Patienten individuelle Möglichkeiten, Fähigkeiten, aber auch Gefahren erkannt und Prognosen gestellt werden. Dazu ist meist eine Fremdanamnese ein wesentlicher Teil der Abklärungen. Das Vorgehen muss innerhalb einer Institution wie auch nach aussen transparent sein und reflektiert werden können, beispielsweise durch Supervision. Behandler sollten Teilhaber der Kultur sein, in der die Patienten leben. Dies bedeutet nicht unbedingt, dass sie «einheimisch» sein müssen, doch sie sollten die Sprache verstehen und die gesellschaftlichen Normen und Regeln kennen und akzeptieren. Sie sollten medizinisch und psychologisch gut ausgebildet sein. Wer Verantwortung hat, sollte einerseits über Erfahrung und andererseits über das notwendige Wissen verfügen. Daneben müssen die Behandler, um den individuellen Patienten gerecht werden zu können, ausreichend Zeit für Diagnostik, Behandlung und Überwachung zur Verfügung haben. Behandlung spielt sich in Beziehungen ab. Diese benötigen Zeit. Da die Arbeit zudem psychisch belastend ist, insbesondere da ja gerade Menschen behandelt werden, die wegen ihres Ver- möglichkeiten mit ausreichend qualifiziertem Personal und guter Vernetzung können helfen, Gewalt und überwältigenden Zwang in der Machtausübung zu verringern. Diesen wechselseitigen Ansprüchen Rechnung zu tragen, ist eine schwierige Aufgabe unserer Gesellschaft, wenn sie sich human und gerecht nennen will, gerade auch bei der aktuellen Problematik des Ärztemangels in der Psychiatrie.

\section{Fazit}

Die Psychiatrie versteht sich einerseits als medizinisch-naturwissenschaftlichen Teilbereich der Medizin. Andrerseits ist sie in unserer Gesellschaft eingebettet in das rechtsstaatliche System und erhält aus ihm Aufträge und Legitimation. Der kranke Mensch in der Psychiatrie kollidiert manchmal durch seine Erkrankung mit Mitmenschen und der Gesellschaft und wird zu einer Behandlung gedrängt oder gezwungen. Dabei gilt nicht wie in der Justiz die Unschuldsvermutung. Allerdings darf nicht vergessen werden, dass primär die Problematik im Umfeld des Menschen mit psychischer Störung besteht und er ja nur deswegen in Behandlung kommt. Erst sekundär, sobald der Kranke in Behandlung ist, wird Machtausübung das Problem der Behandlung. Jenseits aller Fortschritte in der Ursachenforschung und in der Behandlung psychischer Krankheiten besteht für die Psychiatrie dieser Konflikt zwischen Individuum und Gesellschaft. Geeignete, ethisch angemessene Umgangsmöglichkeiten mit dem Machtkonflikt in jedem individuellen Fall wie auch im übergeordnet strukturellen Kontext haben in der psychiatrischen Behandlung einen massgeblichen Anteil am Behandlungserfolg. 